\title{
Kernos
}

Revue internationale et pluridisciplinaire de religion grecque antique

$10 \mid 1997$

Varia

\section{Euenios der Seher von Apollonia und Apollon Lykeios: Mythos jenseits der Texte}

\section{Walter Burkert}

\section{(2) OpenEdition \\ Journals}

Édition électronique

URL : http://journals.openedition.org/kernos/647

DOI : $10.4000 /$ kernos. 647

ISSN : 2034-7871

\section{Éditeur}

Centre international d'étude de la religion grecque antique

\section{Édition imprimée}

Date de publication : 1 janvier 1997

Pagination : 73-81

ISSN : 0776-3824

\section{Référence électronique}

Walter Burkert, «Euenios der Seher von Apollonia und Apollon Lykeios: Mythos jenseits der Texte », Kernos [En ligne], 10 | 1997, mis en ligne le 12 avril 2011, consulté le 22 avril 2019. URL : http:// journals.openedition.org/kernos/647 ; DOI : 10.4000/kernos.647 


\section{Euenios der Seher von Apollonia}

\section{und Apollon Lykeios: Mythos jenseits der Texte}

In jener Wende des Perserkriegs, mit der Herodot sein Werk beschließt, als der Gegenstoß der Griechen sich endgültig gegen Asien wendet, wird der Seher eingeführt, der die Opfer auf Delos leitet: Deiphonos, Sohn des Euenios, aus Apollonia an der Adria ${ }^{1}$. Über diesen Euenios ist eine merkwürdige Geschichte zu erzählen, die Herodot als Exkurs einfügt. Von den militärischen Aktionen hinweg versetzt sie uns gleichsam in eine andere Welt, götternah und doch geheimnisvoll, odysseehaft; von Tieren ist die Rede und von Wundern, vom Ursprung der Sehergabe, und auch von besonderer menschlicher Klugheit:

In Apollonia im Ionischen Meer gebe es "heilige Schafe des Sonnengottes", die tagsüber am Fluß weiden ${ }^{2}$, nachts aber von den reichsten und angesehensten Bürgern bewacht werden, in einer Höhle fern der Stadt. Dort sei einst jenem Euenios die Wache zugefallen; er sei jedoch eingeschlafen, und da drangen Wölfe in die Höhle ein und rissen an die 60 Schafe. Als dies aufkam, wurde Euenios, nach ordentlichem Gerichtsbeschluß der Stadt, zur Strafe geblendet. Doch daraufhin zeigte sich göttlicher Zorn: Die Schafe pflanzten sich nicht mehr fort, die Früchte wuchsen nicht wie zuvor; "offenbart wurde ihnen in Dodona und in Delphi - denn sie fragten die Propheten nach der Ursache des gegenwärtigen Unheils, die aber sagten es ihnen, daß sie zu Unrecht den Wächter der heiligen Schafe, Euenios, des Augenlichts beraubt hatten. Denn sie

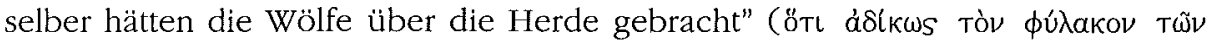

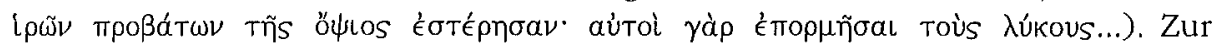

I HDT., IX, 92-96. Kommentare: H. STEIN, Herodotos V, Berlin, 18935; R.W. MACAN, The VII VIII IXth Book of Herodotus, London, 1908; W.W. HOW, J. WELLS, A commentary on Herodotus II, Oxford, $1928^{2}$; A. MASARACCHIA, Erodoto. La Sconfitta dei Persiani. Libro IX della Storia, Verona, 1978. Vgl. P. KETT, Prosopograpbie der bistorischen griecbischen Manteis bis auf die Zeit Alexanders des Grossen, Diss. Erlangen, 1966, S. 38 f.: Nr. 26, Euenios; S. 31: Nr. 18, Deiphonos. Zusätzliche Informationen sind nicht bekannt. Eine Neufassung der Erzählung, evidentermaßen nach Herodot, bringt KONON,

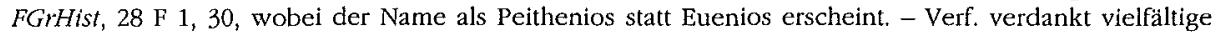
Antegung einem Referat "Euenios the Negligent Watchman" von Alan GRIFFITHS, London anläßlich des Symposiums Myth into Logos? in Bristol, 25. 7. 1996, das in den Akten des Symposiums publiziert wird. Für die hier vorgestellen Rekonstruktionen und Konstruktionen ist der Verf. allein verantwortlich.

2 Die Herodotüberlieferung nennt den Namen des Flusses nicht; Kommentatoren und Editoren haben 'Aoos' (STRABON, S. 271) oder 'Chaon' (THEOGNOSTOS, Canon. 794: Stein) ergänzt. 
Sühne soll Euenios bekommen, was immer er sich wünscht; "sie selbst aber würden dem Euenios ein Geschenk machen, daß viele Menschen ihn ob dieses Besitzes glücklich preisen würden." Die Erzählung konzentriert sich dann darauf, wie klug und geschickt die Apolloniaten das Problem jenes grenzenlosen Wünschens zu lösen wußten; die entscheidende Szene wird von Herodot anschaulich und ausführlich, mit direkter Rede, entfaltet. Ganz knapp heißt es dann, daß Euenios, obschon enttäuscht ob der schlecht genutzten Chance, "danach sogleich eine in seinem Wesen verzwurzelte Seherkunst (É $\mu \phi \cup т{ }^{\prime}$

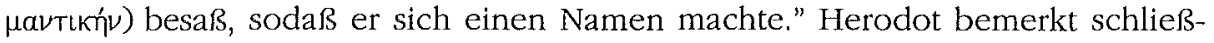
lich noch, es sei gar nicht sicher, ob der Seher Deiphonos wirklich Sohn dieses Euenios gewesen sei, oder nur mit dem berühmten Namen reiste, um sich in

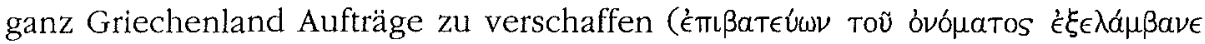

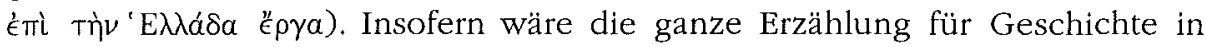
unserem Verständnis doppelt entbehrlich. Aber sie strahlt aus ihrem eigenen Kern.

Man kann die Erzählung in einen mythisch-märchenhaften und einen eher anekdotisch-schwankhaften Teil zerlegen. Dieser ist es, der Herodot besonders interessiert: Wie bringt man den, der alles verlangen könnte, dazu, sich mit relativ Bescheidenem zu begnügen? Über den ersten Teil geht Herodot dagegen mit merkwürdiger Kürze hinweg, so daß der Text Rätsel aufgibt: Welche göttlichen Mächte waren es denn, die 'selber' Wölfe gegen die Schafe des Sonnengottes hetzten, und warum? Man stellt dabei fest, daß Herodots Text

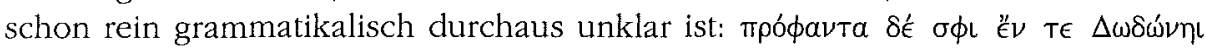

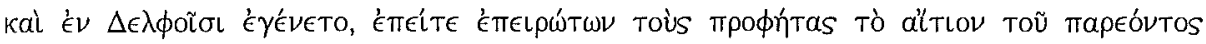

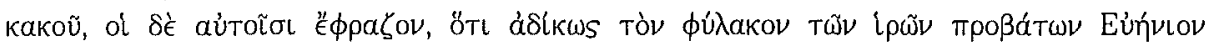

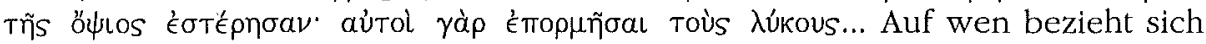
dieses unvermittelt auftretende "sie selber" (aủol)? In der Abfolge der Wörter im

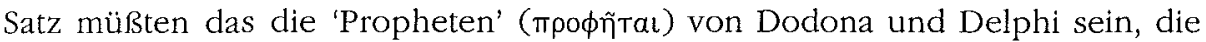
kurz zuvor genannt sind; dies ist offensichtlich absurd. Man hat längst auch

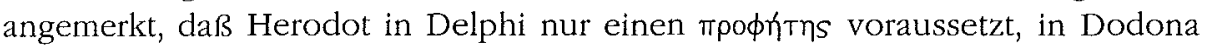
gar keinen, sondern nur Priesterinnen nennt ${ }^{3}$. Darum haben Editoren und Kommentatoren den Text korrigiert, durch Streichungen, über deren Umfang

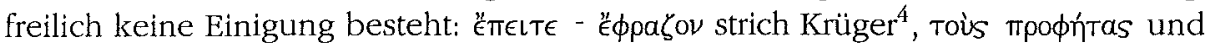

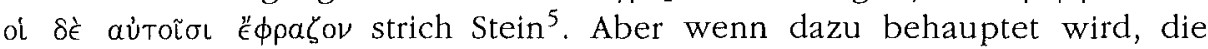
angebliche Interpolation sei ein mißglückter Versuch, jenes aúrol zu erklären, setzen sich die Kommentatoren allzuleicht darüber hinweg, daß nach der

\footnotetext{
3 HDT., VIII, 36, 2; II, 55 (Verweise von STEIN (Anm. 1)).

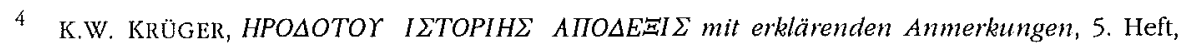
Berlin, 1856: "aus einem ungehörigen Erklärungsversuch eingefälscht".

5 Herodoti Historiae rec. H. STEIN, II, Berlin, 1871, und Komm. z. d. St. Seinem Text folgen C. HUDE (Oxford, $1927^{3}$ [1908]) und HOW-WELLS (Anm. 1); unentschieden MACAN (Anm. 1); gegen Textänderung R. CRAHAY, La littéraiure oraculaire cbez Hérodote, Paris, 1956, S. 82-84, hier S. 83, 1. -

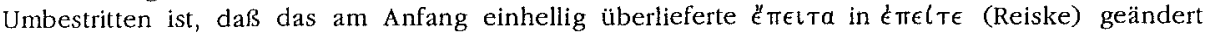
werden mußs.
} 
Streichung die Erklärung dieses Wortes um so mehr in der Luft hängt. Die Götter 'selbst' müßten ja wohl der Gott von Dodona und der Gott von Delphi sein, also Zeus und Apollon, sagt man ${ }^{6}$. Aber das steht nicht im Text; und hat man je gehört, daß Zeus und Apollon gemeinsam Wölfe treiben? Sollten sie dies in getrennten Orakeln bestätigen? Einleuchtend ist die Vermutung, der Ausdruck sei der Sprache von Orakeln nachgebildet: Dort waren dann die Götter 'selbst' genannt $^{7}$ - also etwa in einem Orakeltext des Inhalts: <'Wisset, Leute von Apollonia, daß ihr den Zorn von Göttern auf euch geladen habt. Denn sie selbst...'>. Nun kommt die Gabe der Seherkunst im allgemeinen nicht von Zeus, sondern allein von Apollon; so bekennt es auch Kassandra in der Darstellung des Aischylos ${ }^{8}$. Die Götter selbst, die Wölfe schicken und später die Sehergabe schenken, müßten also dem Kreis des Apollon angehören. Der zweimal verwendete Plural weist allerdings darauf hin, daß Apollon, wenn schon, nicht allein am Werke war; war Leto dabei, und/oder Artemis?

Jedenfalls: Der Herodottext stellt durch andeutende Diktion ein Problem, das von diesem allein aus nicht zu lösen ist. Es mag Interpreten geben, die gerade eine Kunst der Andeutung und Aussparung bei Herodot zu schätzen wissen. Aber syntaktische Unklarheit drängt eher zu der Diagnose: Ein Text mit solchen Bruchstellen ist nicht autonom gestaltet, sondern setzt eine andersartige, ausführlichere 'Quelle' voraus, in der zumindest das Wirken der Götter mit den Wölfen deutlicher gefaßt war, vielleicht mit genauerer Wiedergabe eines Orakeltextes. Herodot ist im ersten Teil seiner Erzählung nicht der souveräne Fabulierer, sondern faßt Vorgegebenes mit Auslassungen zusammen ${ }^{9}$.

Versucht man, die Lücke der Erzählung zu ergänzen, kann man Hinweisen nachgehen, die Name und Lage der Stadt Apollonia liefern. Kein Zweifel: Der Name verbindet die Stadt mit Apollon. Dies ist nicht eine unverbindliche Assoziation, sondern wurde von der Stadt selbst gerade in der Herodot-Zeit ausdrücklich betont: In einer Weihung zu Olympia aus dem 5. Jh. rühmen sich die Apolloniaten, daß "Apollon mit den ungeschnittenen Haaren" ihre Stadt am Meer selbst gegründet hat: Tà

Wenn nun die auf Apollon sich berufende Stadt 'heilige Schafe des Helios' hegt, liegt es nahe die Brücke vom einen zum anderen zu schlagen und Apollon mit Helios gleichzusetzen. Dies haben Kommentatoren angemerkt ${ }^{11}$. Der Text

\footnotetext{
6 So STEIN, danach MACAN, HOW-WELlS, MASARACCHIA (Anm. 1).

7 Crahay, op. cit. (Anm. 5) ; schon STEIN verwies auf das 'Ich' in Orakeln, HDT., I, 47; VII, 141.

8 AISCH., Ag., 1202.

9 Dies ist nicht ohne Bedeutung für die neuerdings verschärfte Debatte um Herodots Zuverlässigkeit; vgl. auf der einen Seite D. FEHLING, Herodotus and bis 'Sources', Leeds, 1979 [Die Quellenangaben bei Herodot, Berlin, 1971], auf der anderen W.K. PRITCHETT, The Liar Scbool of Herodotos, Amsterdam, 1993.

10 PAUS., V, 22, 3; die Inschrift wurde wieder aufgefunden, P.A. HANSEN, Carmina Epigrapbica Graeca Saeculonum VII-V a. Cbr. n., Berlin, 1983, nr. 390.

11 So MACAN, danach MASARACCHIA z. d. St. (Anm. 1).
} 
würde dann zu einem der frühesten Zeugnisse für die Gleichsetzung von Apollon und Sonnengott, neben oder sogar vor Aischylos' Bassariden, die allerdings in einen ganz anderen Zusammenhang weisen ${ }^{12}$. Man könnte aber auch argumentieren, daß die nächtlichen Wölfe, die in die Höhle einbrechen, um Schafe des Helios zu töten, eher als Antithese, als Gegenmacht zum Sonnengott zu verstehen seien. Hier ist vorläufig nicht weiter zu kommen.

Wichtiger ist wohl der andere Hinweis, der sich mit Apollonia an der Adria verbinden läßt: Die Weihegaben der Hyperboreer für Apollon, berichtet Herodot, würden über die Adria nach Dodona und von dort nach Delos gebracht $^{13}$. Und was besonders aufhorchen läßt: Auf dem hyperboreischen Weg seien seinerzeit die hyperboreischen Jungfrauen Arge und Opis "zusammen mit den Göttern selbst" nach Delos gekommen. Die Gaben folgen diesem heiligen Vorbild nach regelmäßigem Brauch ${ }^{14}$. Dieser 'hyperboreische Weg', auf dem Götter wandeln, führt also zumindest in unmittelbarer Nähe an Apollonia vorbei. Daß Apollonia selbst seit alters eine wichtige Station dieses Weges gewesen sei, wurde zuversichtlich behauptet ${ }^{15}$, ist jedoch nicht vereinbar mit dem Text Herodots, wonach die Leute von Dodona die 'ersten der Griechen' sind, die die hyperboreischen Gaben in Empfang nehmen. Daß Beziehungen Apollonia-Dodona bestanden, hat Herodot in der Euenios-Geschichte immerhin angetönt.

Was diese 'hyperboreischen Gaben' für Delos waren und woher sie in Wirklichkeit kamen, ist ein bislang unlösbares Problem. Daß sie in der Tat Delos erreichten, zumindest von Zeit zu Zeit, ist durch Inschriften des 3. Jh. v. Chr. klar bezeugt ${ }^{16}$. Die sogenannten Gräber der 'hyperboreischen Jungfrauen' auf Delos freilich sind, wie man heute weiß, Reste mykenischer Gräber. Sie wurden seit

12 AISCH., Fr. S. 138 Radt; vgl. W. BURKERT, Griechiscbe Religion der archaischen und klassiscben Epoche, Stuttgart, 1977, S. 233.

13 HDT., IV, 33. Zu den 'hyperboreischen Gaben' und ihrem Weg A.B. CoOK, Zeus, II, Cambridge, 1925, S. 494-500; III, 1940, S. 1136 f., der, mit Vorgängern seit F.G. WELCKER, im hyperboreischen Weg die Bernsteinroute findet; C.T. SELTMAN, The Offerings of the Hyperboreans, in CQ, 22 (1928), S. 155-159; R.L. BEAUMONT, Greek Influence in the Adriatic Sea before the Fourth Century B.C., in JHS, 56 (1936), S. 159-204, hier 198 f.; J. TRÉHEUX, La réalité bistorique des offrandes byperboréennes, in G.E. MYLONAS, D. RAYMOND (hrsg.), Studies presented to D.M. Robinson, II, Saint Louis, 1953, S. 758-774; H.W. PARKE, The Oracles of Zeus, Oxford 1967, S. 280-286: "The Hyperborean Gifts". Neben Herodot, IV, 34, stehen Kallimachos, Fr. 186, und Hymn. IV, 278-299, in enger Anlehnung an Herodot; ferner - mit ganz anderer Route - PAUS., I, 31, 2 (man hat als Quelle PHANODEMOS vermutet, vgl. FGrHist, 325 F 2; 29); vgl. auch PLATON, Axiocbos, 371a; PLUT., Mus., 14 (Mor, 1136b); PORPH., Abst., II, 19.

14 HDT, IV, 35, 3.

15 COOK, op. cit. (Anm. 13), II, 499, mit der Vermutung, der Apollonkult in Apollonia sei älter als die Gründung der korinthischen Kolonie (um 600, vgl. BEAUMONT, op. cit. [Anm. 13], S. 168-171). Jedenfalls setzt 'Apollonia' die gemeingriechische, nicht die korinthische Namensform Apellon voraus, die in Korinth (korinth. Aryballos: K. SCHEFOLD, Götter- und Heldensagen der Griechen in der früb-und bocbarchaiscben Kunst, München, 1993, S. 253, Abb. 272), Syrakus (SEG, XXVI, 1118) und Kerkyra (AПE $\Lambda \mathrm{OOOPO} S E G, \mathrm{XXX}, 521)$ noch nachweisbar ist.

16 TRÉHEUX, op. cit. (Anm. 13). 
archaischer Zeit als Zeugen des mythischen 'Dereinst' in Anspruch genommen ${ }^{17}$. Herodot unterscheidet zwei Paare von hyperboreischen Jungfrauen, Hyperoche und Laodike, die ersten Überbringerinnen der Gaben für Eileithyia, denen zu Ehren Jungfrauen vor der Hochzeit und auch die Knaben der Delier sich die Haare scheren, und früher noch Arge und Opis, die "mit den Göttern selbst" gekommen waren. Dies kann sich nur auf die schwangere Leto mit dem noch ungeborenen Apollon, vielleicht auch mit der kurz zuvor geborenen Artemis beziehen. Beide Jungfrauenpaare haben es mit 'Geburt' zu tun, Göttergeburt und Menschengeburten.

Merkwürdigerweise sind die hyperboreischen Jungfrauen aber zugleich mit Lykien verbunden. Denn, sagt Herodot, es war der Lykier Olen, der die Hymnen auf sie gedichtet hat, mit denen man Sammel-Prozessionen in seiner Zeit noch immer durchführt ${ }^{18}$. Wir stehen damit offenbar im Bereich eines Apollon Lykios. Denn die Verbindung von Apollon mit Lykien ist die eine Erklärung, die man in der Antike für den Beinamen Lykios oder Lykeios des Apollon kennt; die andere aber ist der 'wölfische' Apollon ${ }^{19}$. Auf die doppelte Möglichkeit der Interpretation ist zurückzukommen. Sofern der 'Wolf' in Verbindung mit den Hyperboreern und dem hyperboreischen Weg ins Spiel kommt, würde man von anderer Seite wiederum dem nahekommen, was Götter 'selbst' in einer Nacht bei Apollonia der Stadt des Apollon mit Wölfen trieben.

Doch bedarf es keiner Spekulation. Ein ganz anderer Text kommt hier zu Hilfe, der, soweit ich sehe, in keinem Herodotkommentar erwähnt wird. Es geht ja auch vordergründig um etwas ganz anderes, um ein Tiermärchen, einen $\mu$ ÕOos, wie Aristoteles in seiner Tiergeschichte tadelnd sagt, um das ganze zu verwerfen. Die Rede ist von Zeugung und Geburt bei den Wölfen. Dies sei natürlich ähnlich wie beim Hund, stellt Aristoteles fest; "Es wird aber eine Geschichte über die

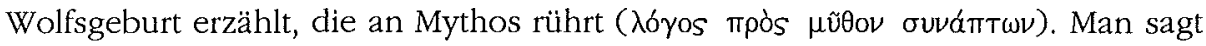
nämlich, daß alle Wölfinnen in zwölf Tagen des Jahres gebären. Die Ursache dafür geben sie in einem Mythos an: In eben so vielen Tagen hätten sie - die Wölfinnen - Leto von den Hyperboreern nach Delos geleitet, wobei Leto sich die Gestalt einer Wölfin gab, aus Furcht vor Hera." Es gibt darüber, sagt Aristoteles, keine systematischen Beobachtungen, man 'erzählt' es nur so eben; falsch sei offenbar auch, was man sich gleichfalls erzählt, daß wölfe nur einmal im Leben gebären ${ }^{20}$.

17 Siehe Ph. Bruneat, J. DuCAT, Guide de Délos, Paris, 1965, S. 94 (nr. 32); S. 97 f. (nr. 41). Die Ankunft Apollons mit den hyperboreischen Jungfrauen ist vielleicht auf der bekannten kykladischen Amphora dargestellt, die wohl ein Weihgeschenk für Delos war, SCHEFOLD, op. cit. (Anm. 15), S. 61, Abb. 39.

18 HDT., IV, 35, 3-4.

19 Den Beinamen Lykeios im Zusammenhang des Wortstammes 'Licht' zu verstehen, wurde im 19. Jh. populär.

20 ARISTT., Hist, an., 580a 14-23. 
Hier treffen wir wiederum den Weg von den Hyperboreern nach Delos im Zusammenhang mit der Apollon-Geburt, den auch Herodot kennt; hier aber handelt es sich ausdrücklich um einen Weg der Wölfe; er muß, gemäß jenen anderen Angaben, über die Adria und über Dodona führen, vorbei an Apollonia. Die Wölfinnen sind die Geleiterinnen der Göttin, in deren Mitte sie sich verbirgt, und offenbar zugleich trächtig wie die Göttin selbst. Die Reise dauert zwölf Tage; und diese Ordnung der Zeit hat sich fortgesetzt: In dieser Periode, und nur in ihr, werfen alle Wölfinnen seither ihre Jungen. Nimmt man dies mit Herodots Erzählung von Euenios zusammen, wird klar, was in bestimmten Nächten des Jahres den Wölfen das Recht über die Schafe gibt, und seien es die des Sonnengottes: Die Wolfsnacht hat ihren göttlichen Ursprung. Was Herodot in abgebrochenem Stil andeutete, ist damit erklärt: 'Die Götter selbst' geben zu gewisser Zeit den Wölfen freie Bahn selbst gegen heilige Schafe, und niemand darf sie hindern..

Der Mythos, daß Leto als Wölfin geboren habe, ist auch Aelian bekannt ${ }^{21}$. Doch gibt es über den Weg der Leto nach Delos zu Apollons Geburt bekanntlich konkurrierende Texte, die weit prominenter geworden sind: Anders erzählt der Apollonhymnus die Wanderung der Leto, anders Kallimachos ${ }^{22}$, keiner der beiden bringt Hyperboreer oder Wölfe ins Spiel. Auch können wir uns die hyperboreischen Jungfrauen, von denen Herodot spricht, kaum als Begleiterinnen der Wolfsmeute vorstellen. Ist also noch eine weitere Variante von Letos Wanderung zu entwerfen?

Mythen existieren in Varianten und treten je nach ihrer Verwendung verschieden auf. Und doch können getrennte Texte gemeinsam auf einen eigentümlichen Mythos zurückverweisen. In unserem Fall liegen zwei Texte vor, die literarisch nichts miteinander zu tun haben: Auf der einen Seite die Legende vom blinden Seher bei Herodot, verknappend und doch erzählfreudig, auf der anderen Seite ein Tier-Aition, das der davon berichtende Aristoteles als Fabelei entlarvt. Und doch ergänzen sich beide Texte zu einem bildhaft geschlossenen, packenden Mythos. Dabei bringt die Geschichte aus Apollonia eine dramatische Episode, sie setzt andeutend die heilige Wolfsreise voraus und gibt einen geographischen Anhaltspunkt an der Adria; die Tierfabel stellt die göttliche Reise vor Augen, nennt die Ordnung der Zeit und behauptet die Fortwirkung im Bereich der wilden Natur bis auf die Gegenwart. Wenn es denn nochmals zu beweisen wäre: Mythos ist nicht mit einem bestimmten Text gleichzusetzen, Mythos ist ein Fundus, der in ganz verschiedenen Texten verarbeitet und zum Ausdruck gebracht werden kann.

Dem Mythologen bleibt der Eindruck, den so gewonnenen Mythos noch keineswegs hinreichend entschlüsselt zu haben. Undeutlich ist, wo der 'Sitz im

21 AEL., Nat. an., X, 26; etwas anders Schol. T $1 l$., IV, 101: ein Wolf führt Leto nach der Geburt nach Xanthos. Zu Apollon und Wolf, W. BURKeRT, Homo Necans, Berlin, 1972, S. 138. Vgl. zu Apollon, Wölfen und Lykien auch XANTHIOS und NIKANDROS bei ANTON. LIB., 35.

22. HOM., Hymn. Apoll., 30-90; KALLIM., Hymn. IV, 51-205. 
Leben' dieses Mythos zu suchen ist, in Apollonia in Illyrien, auf Delos, oder etwa in der persönlichen Legende eines Mantis? Von Apollonia wissen wir weiter so gut wie nichts, abgesehen von der Berufung auf Apollon den Gründer in jenem Epigramm. Fassen wir Delos ins Auge, den Ort der Geburt Apollons nach aller Überlieferung, so bleiben wir mit den divergierenden Varianten von Letos Wanderungen konfrontiert. Vor allem kommt jene auffällige Doppeldeutigkeit ins Spiel, die mit Apollon Lykeios oder Lykios verbunden ist: Verweist der Name auf das kleinasiatische Lykien oder aber auf den Wolf? Oder müssen wir sogar anerkennen, daß Götter-Beinamen eine grundlegende Ambiguität enthalten können, die von Fall zu Fall von Dichtern und Deutern benützt und in einer bestimmten Weise festgelegt wird? Die Beziehung Apollons zu Lykien ist bereits in der Ilias vorgegeben ${ }^{23}$, wird von Herodot betont ${ }^{24}$, hat in der neueren Forschung zur These von kleinasiatischen 'Ursprung' des Apollon geführt ${ }^{25}$; aber im Vordergrund stand für die Griechen immer wieder, in Argos und besonders auch in Delphi, der Wolf-Apollon ${ }^{26}$. Denkbar ist, daß in einer bestimmten Situation auf Delos Anlaß bestand, die Wolfs-Assoziation ins Spiel zu bringen; denkbar wäre auch, daß man beide Orte, Apollonia und Delos, in Verbindung bringen wollte, indem jenes zu rekonstruierende Orakel die Apolloniaten über den hyperboreischen Weg und das Recht der Wölfe belehrte, wovon sie offenbar nichts gewußt hatten. Näher liegt es, die Legende an die Mantis-Familie gebunden sein zu lassen, auf die Herodot verweist: Deiphonos habe sich gleichsam als Trittbrettfahrer vom Ruhm des Euenios tragen lassen, was doch heißen muß: Deiphonos selbst hat sich auf diese Geschichte berufen.

Seher pflegen in der Tat zum eigenen Ruhm sich an 'Vorfahren' zu halten, mythische Ahnen zumal; auf jeden Fall bedarf es der Familientradition ${ }^{27}$. Herodot berichtet ausführlich von Teisamenos, dem Melampodiden, der sich bemüßigt fühlte, in seinen Forderungen gegenüber Sparta seinen berühmten Ahnen nachzuahmen ${ }^{28}$. Doch läßt sich konkreter werden:

Deiphonos leitet nach Herodots Bericht eben auf Delos die Opfer der Griechen, in dem Augenblick, als der Vorstoß nach Osten beginnt ${ }^{29}$; seine

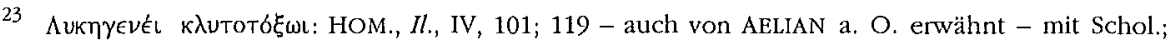
wahrscheinlich ist der Troer Pandaros (Il., II, 827) zum Lykier (Il., V, 173) geworden durch eine

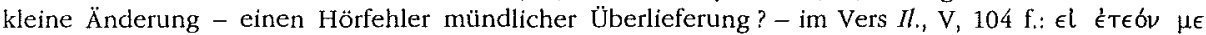

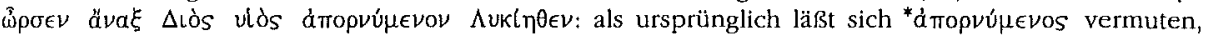

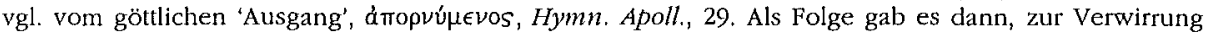
antiker Geographen und moderner Historiker, Lykier in der Troas. Vgl, auch P. FREI, Die Lykier bei Homer, in Proceedings of the Xtb international Congress of Classical Archaeology, Ankara, 1978, S. 819-827.

24 Vgl. bei Anm. 18.

25 U. v. WilamoWitz-MOEllendorfF, Apollon, in Hermes, 38 (1903), S. 575-586; dazu W, BURKERT, Apellai und Apollon, in RhM, 118 (1975), S. 1-21, bes. S. 1-4.

26 Zu den Delphern als 'Wölfen' und zur Lykoreia-Tradition: BURKERT, Homo Necans, S. 138.

27 Vgl. W. BURKERT, The Orientalizing Revolution, Cambridge, Mass., 1992, S. 41-46.

28 HDT., LX, 33-36.

29 HDT., LX, 92, 2; 96, 1. 
Verkündigung des $\kappa a \lambda \lambda \iota € \epsilon \tilde{\nu} y$ garantiert den Erfolg. Dies macht Deiphonos zum 'Sieger' in diesem Feldzug, so gut wie sein Kollege Teisamenos fünf 'Siege' errang, darunter den von Plataiai (IX, 35, 2) ${ }^{30}$. Und auch Deiphonos der 'Sieger' hat nun seinen Mythos. Pindar hat anläßlich eines andersartigen Siegs in seine 6. Olympische Ode den Mythos von Iamos, dem Ahnherrn der Iamiden von Olympia aufgenommen. Auf Delos, beim entscheideneden Auszugs-Opfer oder vielleicht eher in der Wiedererinnerung anläßlich einer nachträglichen Erinnerungs- und Siegesfeier auf Delos nach dem glücklichen Ausgang - hat die Geschichte von Euenios zu Ehren des Deiphonos ihren Ort. Man mag dabei bedenken, daß, was Apollon Lykeios oder Lykios betrifft, in dieser Situation im Jahr 479 der Bezug auf Lykien nicht recht am Platze war, gehörte Lykien doch zum Feindesland, gegen das die Speerspitze des Eroberers gerichtet war; umso eher mochte es sich anbieten, die nördlichen Aspekte eines 'wölfischen' Apollon lebendig zu machen. Man kann demnach vermuten, daß die wölfische Variante des hyperboreischen Wegs in diesem Zusammenhang ausgestaltet und verbreitet wurde. Ob ein Dichter zugegen war, der den Deiphonos so wie Pindar den Iamiden feierte, bleibt unserer Phantasie überlassen.

Von zwei Texten ohne rechten Kontext aus sind wir zum einleuchtenden 'Sitz im Leben' eines Mythos vorgedrungen, ohne doch einen damals aktuellen Text zu kennen. Dies heißt jedenfalls auch nicht, daß der Mythos genau bei dieser Gelegenheit frei erfunden wurde. Gewiß, wir sehen die Elemente namentlich aus der Odyssee, aus denen die Euenios-Geschichte aufgebaut ist die Sonnenrinder und -schafe der Odyssee ${ }^{31}$, auch die Kyklopenhöhle mit ihren Schafen, auch die Göttergabe für den, dem das Augenlicht genommen ist ${ }^{32}$, dazu der hyperboreische Weg über die Adria; doch eben der von Herodot weitergegebene Zweifel, of Deiphonos überhaupt in Wahrheit der Sohn des Euenios gewesen sei, setzt den Ruhm eben dieses Mantis unabhänig von Deiphonos und vor dessen Karriere voraus.

In der Tat scheint der Mythos von Leto der Wölfin und der Geburt des Apollon Lykeios noch weitere Hinweise zu enthalten, die nach Deutung verlangen und doch frustrierend unbestimmbar bleiben. Da sind einmal die 'zwölf Tage' der hyperboreischen Reise. Sie entsprechen keiner menschlichen Reisezeit - schon von Epidamnos nach Apollonia reiste ein Mensch zwei Tage -, sie sind gewiß nicht von der Natur im Jahreslauf festgelegt. Es muß sich um ein kulturell etabliertes Datum handeln, ein Festdatum geradezu - ohne daß es gelingen will, dies mit einem sonstigen Zeugnis zu kombinieren oder an einem

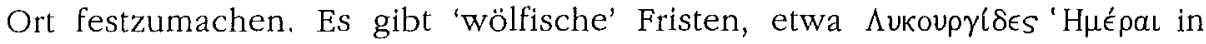

30 HDT., IX, 35, 2.

31 Seit Stein verweist man auf Od., XII und Hymn. Apoll., 412. Zu weiteren Tieren des Jenseits: W. BURKERT, Structure and History in Greek Mytbology and Ritual, Berkeley, 1979, S. 83-94; SacrificioSacrilegio: Il Trickster fondatore, in Studi Storici, 25 (1984), S. 835-845 = C. GROTTANELLI, N.F. PARISE (hrsg.), Sacrificio e società nel mondo antico, Bari, 1988, S. 163-175.

32 Od, VIII, $63 \mathrm{f}$. 
Sparta ${ }^{33}$; die 'Wolfsabwehrer' im Namen lassen aufhorchen, doch sind keine Einzelheiten bekannt; der Name des angeblichen Gesetzgebers Lykourgos hat hier alle weiteren Informationen unterdrückt. Man könnte auch den thrakischen Lykourgos nennen, der zu seinem Unheil die rasenden Begleiterinnen des Dionysos abzuwehren versucht; doch bedarf es zu vieler hypothetischer Substitutionen, um dies mit dem Mythos von Apollonia zuzsammenzubringen. Assoziationen mit den zwölf Rauhnächten zwischen Weihnachten und Epiphanias liegen nahe, bringen aber bestenfalls eine strukturelle Parallele. Näher noch lägen die Tage der lustratio um die römischen Lupercalia ${ }^{34}$ wieder eine Periode, die auf 'Wölfe' verweist, und doch schon zu weit entfernt, um fürs Griechische einen Beitrag zu leisten.

Auch nach einer Bedeutung der '60' getöteten Schafe kann man fragen, ohne recht weiter zu kommen. Herodot teilt uns nicht mit, wie groß die Herde des Sonnengottes war. Man hat sich immer an die Sonnenherden der Odyssee erinnert; diese bestehen aus 350 Tieren ${ }^{35}$, die Zahl sollte unverändert bleiben und schon Aristoteles wird für die These zitiert, daß dies den Tagen des Jahres entspreche, was freilich nur sehr grob stimmen würde. Immerhin wurde vermutet, daß auch die Herde von Apollonia aus 350 oder vielleicht 360 Schafen bestand $^{36}$. Sollte der Verlust also zwei Monaten entsprechen, zwei Wintermonaten des 'wölfischen' und gerade nicht des lichten Apollon? Apollon und die Ordnung der Zeit scheinen vielfältig zusammenzuhängen ${ }^{37}$. Aber hier bauen wir ins Leere.

Walter BURKERT

Klassisch-Philologisches Seminar der Universität Zürich

33 PLUT., Lyk, 31, 9.

34 Zu den Lupercalia Chr. ULF, Das römiscbe Lupercalienfest, Innsbruck, 1982 und W. PÖTSCHER, Die Lupercalia. Eine Strukturanalyse, in $G B, 11$ (1984), S. 221-249 = Hellas und Rom, Hildesheim, 1988 , S. $517-546$.

35 Od., XII, 128-131; Schol. zu 128/129 und EUSTATH. 1717 mit Verweis auf ARISTOTELES (Fr. 175 Rose).

36 STEIN z. d. St. : "nach den Jahrestagen, denen sie entsprechen", danach HOW-WELLS (Anm. 1).

37 M.P. NILSSON, Die Entstebung und religiöse Bedeutung des griecbischen Kalenders, Lund, $1962^{2}$. 